\section{PSICOLOGIA IBEROAMERICANA}

\section{Psicología lberoamericana}

ISSN: 1405-0943

psicología.iberoamericana@uia.mx

Universidad Iberoamericana, Ciudad de

México

México

Hernández, Erika Berenice; Contreras Ibáñez, Carlos César

Motivación al Logro, Autoeficacia, Expectativas de Vida y Orientación Cultural Como Determinantes

de Bienestar Subjetivo en Adoescentes de la Ciudad de México

Psicología Iberoamericana, vol. 13, núm. 1, 2005, pp. 48-57

Universidad lberoamericana, Ciudad de México

Distrito Federal, México

Disponible en: http://www.redalyc.org/articulo.oa?id=133926982008

Cómo citar el artículo

- Número completo

- Más información del artículo

- Página de la revista en redalyc.org

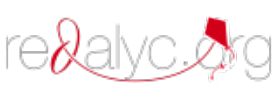

Sistema de Información Científica

Red de Revistas Científicas de América Latina, el Caribe, España y Portugal

Proyecto académico sin fines de lucro, desarrollado bajo la iniciativa de acceso abierto 


\title{
Motivación al Logro, Autoeficacia, Expectativas de Vida y Orientación Cultural Como Determinantes de Bienestar Subjetivo en Adolescentes de la Ciudad de México Achievement Motivation, Self-Efficacy, Life Expectations and Cultural Orientation as Determinants of Subjective Well-Being in México City Adolescents
}

\author{
Erika Berenice Hernández y Carlos César Contreras Ibáñez* \\ Universidad Autónoma Metropolitana
}

\section{Resumen}

En un nivel de análisis individual, la satisfacción con la propia vida está vinculada a la diferentes variantes constitutivas del self, mientras que en el nivel colectivo se asocia con los valores culturalmente establecidos. El presente estudio explora la relación entre las expectativas de vida, autoeficacia, motivación al logro y el colectivismo-individualismo como determinantes del bienestar subjetivo en adolescentes de entre 12 y 19 años de edad, estudiantes de la Ciudad de México. Participaron en este estudio 450 adolescentes, los cuales fueron seleccionados a través de su escolaridad y ocupación laboral. Se aplicó un instrumento con 190 reactivos que contenía diversas escalas que miden las variables antes descritas para los ámbitos general, familiar, escolar, laboral y de pareja. Los resultados muestran cómo la percepción de bienestar influye en la elaboración de las expectativas individuales en función de lo que se "tiene" y lo que se "espera lograr".

Descriptores: bienestar subjetivo, expectativas vitales, autoeficacia, motivación al logro, individualismo-colectivismo, adolescentes

\begin{abstract}
In an individual level of analysis, the own life satisfaction is linked to distinct self constitutive variants, while at collective level, with the culturally established values. The present study explores the relationship between expectations of life, self-efficacy, achievement motivation and of collectivism-individualism, as determinants of subjective wellbeing among adolescents (12-19 years old) studying in Mexico City. Four hundred fifty adolescents participated in this study, seiected through their schooling and work occupation, responding an instrumen with 190 items, contained in diverse scales measuring the variables before described for general, familiar, school, job and coupling spheres. Results show how perception of well-being influences the elaboration of individual expectations as a function of what you "have" and "hope to achieve."

Descriptors: subjective well-being, life expectations, self-efficacy, achievement motivation, individualismcollectivism, adolescents
\end{abstract}

Últimamente se ha vuelto moneda corriente emplear el término calidad de vida en muy variados contextos. Así, políticos, economistas, sociólogos y psicólogos, entre otros, lo usan indistintamente, al tiempo que representa una premisa o aspiración que da sustento a todo tipo de discursos. El mismo constituye un terreno donde varios especialistas aportan diversos enfoques (Grau, 1998). Desde el enfoque psico-

- Dirigir correspondencia a: Universidad Autónoma Metropolitana, Iztapalapa. Cubículo H-160. Avenida San Rafael Atlixco 186, Col. Vicentina, México D.F., C.P. 09430. Tel. 58044790 . Correo electrónico: ccci@xanum.uam.mx 
social, este concepto ha sido precisado como bienestar subjetivo y hace referencia principalmente al modo en que las personas perciben y evalúan su vida como un todo. Su estudio incluye tanto factores externos (Settersten y Mayer, 1997) como características internas a las personas (Costa y McCrae, 1980) como principales predictores de la satisfacción con la vida.

Los componentes principales de este concepto son la satisfacción con la vida y el balance afectivo (Diener, 2003), lo que quiere decir que el bienestar subjetivo debe estudiarse desde el punto de vista afectivo o emocional, considerando el equilibrio entre el afecto positivo y negativo, y desde el punto de vista cognitivo, entendido como la valoración que la persona hace de su vida general o de aspectos parciales de ésta $(\mathrm{Pa}-$ lomar y Pérez, 2001).

El estudio del bienestar subjetivo nos remite a aquellos ámbitos importantes en los que se desarrolla la vida de una persona. Sin embargo, no todos los ámbitos tienen el mismo peso específico en la valoración global del bienestar subjetivo, ni tampoco se puede establecer un orden de importancia, ya que su relevancia varía en cada persona (López y Páez, 1996). Por el hecho de ser una evaluación personal no puede prescindir de la situación concreta del sujeto; cada individuo debe ser considerado como la persona más capaz de sopesar satisfacciones e insatisfacciones con su vida, a pesar de que dos personas se encuentren en una misma circunstancia socioeconómica (Grau, 1998). Por ello, la forma para determinar el grado de bienestar que las personas tienen en su vida, se basa en los juicios de satisfacción o insatisfacción como una evaluación global, o bien pueden estar relacionados sólo a determinadas facetas de la misma. Así, aspectos como el familiar, el escolar, el de trabajo o el de relación de pareja no tienen el mismo peso en un individuo, ya que puede encontrarse satisfecho con su relación familiar pero muy insatisfecho con el trabajo que desempeña.

Algunos estudios relacionados con el bienestar subjetivo han considerado a las diferencias individuales como variables predictoras de la satisfacción personal. Valenzuela, Díaz-Loving y Manjárrez (1996) afirman que la relación entre el sexo y el bienestar subjetivo de las personas es contradictoria. Por una parte, las mujeres reportan insatisfacción en la vida más frecuentemente que los hombres, mientras que en otros estudios, por el contrario, son ellas las más satisfechas. Ante estos hallazgos, algunos autores concluyen que las diferencias entre los sexos se deben a la inten- sidad con la que se viven las experiencias; la mujer, generalmente, las vive más intensamente, debido a que el balance afectivo se centra en las experiencias emocionales de la vida diaria más que en la valoración cognitiva.

Por otra parte, en estudios realizados a partir de la perspectiva cognitivo-motivacional, se ha comenzado a considerar a los planes o metas que se plantean las personas como determinantes del bienestar subjetivo, ya que es el logro o no de las metas personales lo que se relaciona directamente con el bienestar psicológico (Díaz y Sánchez-López, 2001). Dichos estudios reportan que las metas personales, consideradas como unidades mediadoras, proporcionan información, no sólo sobre lo que la persona "es o tiene" sino lo que la persona "hace o espera lograr" (Cantor, Norem y Brower, 1987, citados por Díaz y Sánchez-López, 2001).

Si partimos de la definición que algunas teorías psicológicas realizan de las expectativas, podemos afirmar que éstas son consideradas como uno de los principales determinantes de la conducta y que son definidas como una evaluación subjetiva de la probabilidad de alcanzar una meta concreta (Muneé, 1989). Mientras que el bienestar subjetivo permite hacer una valoración del impacto psicológico de la diferencia entre lo que la persona tiene y lo que espera tener o cree merecer, las expectativas vitales serian justamente esa especificación de cómo se visualiza estar o ser.

El concepto de autoeficacia se refiere, en un sentido amplio, a las creencias que las personas tienen acerca de sus propias capacidades para el logro de determinados resultados (Bandura, 1999). La autoeficacia es un concepto que puede ser usado en un contexto específico. Cuanto más fuerte sea la autoeficacia percibida, más retadores son los objetivos y metas que se establecen las personas y, por tanto, su compromiso es más firme para alcanzarlos (Bandura, 1986). Por tanto, en este estudio suponemos que la variable de autoeficacia está relacionada con el bienestar subjetivo y ésta a su vez con las expectativas generales. Suponemos que las creencias de los adolescentes en relación a su eficacia personal para manejar las demandas vitales influyen sobre su satisfacción personal.

Otra variable relacionada de personalidad es la motivación al logro, definida como el resultado del conflicto emocional entre la búsqueda de los éxitos y el temor a los fracasos sobre el resultado de una conducta particular. McClelland (1985) sostiene que las personas con un historial de éxitos siguen esforzán- 
dose por conseguir nuevos éxitos; sin embargo, aquéllas que han experimentado constantes fracasos (o al menos así lo creen) trabajan más bien para evitar nuevos fracasos.

En la presente investigación consideramos la motivación al logro como un factor importante, porque podemos esperar que los jóvenes hayan tenido éxito en controlar los acontecimientos importantes de su vida y mantener mayores esperanzas o expectativas, y pensarán que si desean alcanzar un objetivo deberán esforzarse para conseguirlo.

Éstas son algunas variables de personalidad que consideramos implicadas con el bienestar subjetivo; sin embargo, debemos considerar también que la cultura moldea a las personas, ya que las personas se encuentran inmersas en ella.

Las culturas se diferencian, siguiendo a Triandis (1994), de acuerdo con el énfasis que hacen sobre el individuo o la colectividad. Aunque las personas tienen tanto cogniciones individualistas como colectivistas, la probabilidad de mostrar una de estas modalidades depende de la cultura misma (Tapia, 1998). En términos generales podemos decir que el concepto de individualismo-colectivismo es un término que estudia los intereses individuales y colectivos de las personas. El individualismo enfatiza una autonomía personal, mientras que el colectivismo agrupa los valores que enfatizan la dependencia del individuo con los grupos con los que se identifica, o bien, a los que pertenece (Triandis, 1990). Esta variable ha sido relacionada con el bienestar subjetivo (Diener, 2001)

Campbell, Converse y Rodger (1976) encontraron que los jóvenes se encuentran más felices que los ancianos, pero que a menudo también manifiestan estar más insatisfechos. Resulta importante conocer la percepción que los jóvenes tienen de su vida actual, qué tan satisfechos o insatisfechos están actualmente sus propias expectativas de futuro, y qué relación guardan éstas con su bienestar subjetivo presente.

Los jóvenes en México, al igual que los de la mayoría de los paises latinoamericanos, se ven afectad por las políticas de ajuste macroeconómico aplicadas por los gobiernos. Dichas políticas, de índole económica, política y social, llegan a afectar en ocasiones el bienestar psicológico y social de los jóvenes; incluso llegan a verse afectados sus logros y expectativas personales. Estas políticas causan, en ocasiones, un difícil presente, en el que el futuro resulta desalentador incluso para el más preparado de los adultos y, con más razón aún, para los jóvenes de nuestra sociedad.
Las expectativas vitales de los jóvenes mexicanos al inicio del siglo son básicamente estudiar, trabajar y casarse (Instituto.Mexicano de la Juventud, 2000); nos damos cuenta que las expectativas condicionan en algún sentido el futuro. El hecho de elegir un trabajo, una carrera, una pareja, etc., permite que los jóvenes se comprometan con ellos mismos.

En síntesis, esta investigación tiene varios objetivos: 1 . Desarrollar y validar una instrumento de aplicación sencilla para conocer el nivel de expectativas, tanto en los ámbitos familiar, escolar, laboral y de relación de pareja, como en general, que tienen jóvenes en situación escolar de la ciudad de México. 2. Medir de manera concurrente a lo anterior, la autoeficacia, la motivación al logro y el bienestar subjetivo en los cinco ámbitos enunciados, así como la orientación cultural (colectivismo-individualismo) en una muestra que incluya adolescentes que estudien bachillerato propedéutico o técnico-terminal. 3. Conocer si las variables enunciadas en el objetivo anterior explican el bienestar subjetivo, y si hay un efecto moderador del sexo en esta relación.

Nos enfocamos a la población joven, específicamente adolescente, dadas las difíciles condiciones en las que se encuentran a partir de los cambios socioeconómicos que experimenta el país, las cuales condicionan y restringen las decisiones que tienen que tomar en torno a su futuro. Además, consideramos relevante tomar en cuenta el tipo de sistema escolar al que se asiste debido a que, claramente, las escuelas que preparan a los jóvenes para continuar sus estudios parten de la premisa de que ello es posible y les fomenta un proceso de maduración distinto a quienes asisten a estudios dirigidos a la incorporación inmediata al mercado de trabajo (ANUIEs, 2000). Finalmente, diversos estudios indican que el sexo, en su elaboración identitaria como género, condiciona fuertemente lo que se espera hacer en la vida (Affleck et al., 1989).

\section{Método}

\section{Participantes}

Para llevar a cabo la presente investigación se contó con la participación de 450 adolescentes, 300 de ellos estudiantes de nivel medio básico y medio superior y 150 jóvenes que se encontraban recibiendo una instrucción técnica (por ejemplo, cursos de cómputo, cultura de belleza, entre otros; en general, cursos que les 
permitieran ingresar al mercado de trabajo más fácilmente). La edad de los adolescentes oscilaba entre los 12 y los 19 años, con una media de 16 años y una desviación estándar de 2.26 . El $57 \%$ de los participantes fueron mujeres y el resto hombres. El muestreo fue no probabilístico, intencional, a partir del rango de edad definido para esta investigación como adolescente (12 a 19 años), por cuotas de tipo de escuela ( $2 / 3$ de bachillerato propedéutico y $1 / 3$ técnico ó terminal).

\section{Instrumentos}

Se empleó un cuestionario que incluia las escalas correspondientes a cada variable de interés; los datos de validez y confiabilidad observados, asi como sus estadísticos muestrales se muestran en la sección de resultados.

Para evaluar bienestar subjetivo se empleó la escala propuesta por Diener (2003), en el aspecto cognoscitivo. La escala original incluia áreas específicas, tales como ámbito general, familiar, escolar y de relación de pareja. En esta investigación, se retomaron estas mismas, además de incluir una referente al ámbito laboral. Para cada ámbito se tenían cinco reactivos, los cuales fueron redactados de acuerdo a la muestra y al objetivo de la investigación; ejemplos de los ítemes son: "Si pudiera vivir mi vida de nuevo, no cambiaria nada". "La mayoria de los aspectos de mi vida está cerca de mi ideal". "Estoy satisfecho con mi vida". "Hasta ahora he conseguido las cosas importantes que he querido en la vida" y " $\mathrm{La}$ situación actual de mi vida es excelente". Las opciones de respuesta tuvieron un rango entre 1 (Totalmente en desacuerdo) 7 (Totalmente de acuerdo).

Para medir expectativas se creó un instrumento (véase anexo) con una estructura en la que cada ámbito incluyó seis reactivos sobre aspectos importantes para los jóvenes, obtenidos de entrevistas previas. Las opciones de respuesta iban desde 1 (intensidad menor) a 4 (intensidad mayor), en relación con el aspecto que se esperaba medir.

Por su parte, la escala de autoeficacia la integraron 32 ítemes. Los reactivos fueron elaborados a partir de juicios de capacidad, tomando en cuenta las áreas antes mencionadas y una situación en particular. Algunos reactivos empleados fueron: "Me considero lo suficientemente capaz de cumplir las metas que me proponga en la vida; o bien, "Creo que ten- go la capacidad de hacer bien las cosas eñ mi trabajo"; por mencionar algunos ejemplos.

En cuanto a la escala de motivación de logro, se definieron 23 itemes que describian metas u objetivos específicos para cada ámbito. Cada meta fue calificada en términos de su importancia subjetiva con opciones de respuesta que iban de 5 (Sumamente importante) a 1 (Nada importante). Esta escala estuvo sujeta a prueba y su empleo en este estudio simplemente explora su rol como predictor y covariable.

Para medir individualismo-colectivismo se empleó la escala empleada por Correa, Contreras-Ibáñez, Ramírez y López (2002), que consiste en 47 reactivos que operacionalizan orientaciones sociales colectivistas (las metas y necesidades del grupo son igual o más importantes que las personales) e individualistas (lo individual precede a lo grupal), y que es evaluada en una escala de nueve puntos (1 Total desacuerdo a 9 Total acuerdo).

Al final del instrumento se tuvieron preguntas como edad y sexo, las cuales fueron también empleadas en esta investigación.

\section{Procedimiento}

Se acudió a diversas secundarias y escuelas correspondientes al nivel medio superior y, con previa autorización de los profesores, se aplicó el cuestionario en los salones de clases. La población fuera del bachillerato propedéutico fue localizada en escuelas técnicas (cultores de belleza, mecánicos, técnicos en refrigeración, etc.) y en espacios abiertos, solicitando a los participantes su colaboración, asegurándoles que sus respuestas serían anónimas. El tiempo aproximado para responder el cuestionario fue de 40 minutos.

Para el análisis de los resultados se utilizó el paquete SPSS versión 10.0 a fin de obtener la estadística descriptiva, análisis factoriales y correlaciones correspondientes a cada escala, así como un análisis de regresión lineal que permitió construir un modelo de tipo causal de la percepción de satisfacción con la vida de los adolescentes.

\section{Resultados}

A partir del análisis factorial realizado (componentes principales, rotación varimax, normalización de Kai- 
ser) a cada escala obtuvimos lo siguiente: la escala de bienestar subjetivo general, explicó el $50.1 \%$ de la varianza total de sus cinco reactivos; su confiabilidad era de .72, su media de 2.36 y su desviación estándar de 3.42. La segunda correspondió al bienestar subjetivo familiar, que explicó el $66.3 \%$ de la varianza, con una media de 2.35 , una desviación estándar de 4.24 y confiabilidad de .87 . El tercer factor, bienestar subjetivo escolar, logró explicar el $52.9 \%$ de la varianza, con una media de 2.26 , desviación estándar de 3.55 y una confiabilidad de .769 . El cuarto factor correspondió al bienestar subjetivo en el trabajo, que explicaba el $53.43 \%$ de la varianza, con una media de 2.44 y desviación estándar de 4.14 ; su confiabilidad fue de .776. Finalmente, obtuvimos la subescala de bienestar subjetivo en la relación de pareja, factor que explicó el $60.10 \%$ de la varianza, con una media de 2.67 , desviación estándar de .830 . y una confiabilidad de .591 .

En la escala de expectativas obtuvimos también cinco subescalas. La primera de ellas, expectativas generales, la conformaron las preguntas 1 a la 4 (véase anexo) y logró explicar el $41.12 \%$ de la varianza y un valor propio de 2.05 ; su media fue de 3.31 , la desviación estándar de 2.02 y una confiabilidad de .532 . El segundo factor, expectativas familiares, lo integraron las preguntas 6 a la 11 ; la varianza explicada de esta subescala fue de 30.67 y un valor propio de 1.84; la media fue de 3.44 y la desviación estándar de 1.72 y logró una confiabilidad de .614. Las preguntas 12 a la 15 conformaron el tercer factor, bienestar subjetivo escolar; obtuvo un valor propio de 1.94 y explicó el $48.73 \%$ de la varianza; su media fue de 3.45 y desviación estándar de 1.70; su confiabilidad fue de .642. El cuarto factor, bienestar subjetivo en el trabajo, lo integraron las preguntas 16 a la 21 ; este factor explicó el $44.23 \%$ de la varianza; obtuvo un valor propio de 2.65 y una confiabilidad de .723 . El último factor de esta escala correspondió al formado por las preguntas 22 a la 27 , las cuales se referian al bienestar subjetivo en la relación de pareja; logró explicar el $22.36 \%$ de la varianza y obtuvo un valor propio de 1.34 , una media de 3.78 , desviación estándar de 1.03 y una confiabilidad de .591 .

En el caso de las demás escalas también se obtuvieron subescalas. Y en el caso de la escala de individualismo-colectivismo sólo resultaron importantes para esta investigación los siguientes tres factores: egoismo, independencia e individualismo. La tabla 1 muestra las características de las mismas.
Tabla 1. Escalas y subescalas del instrumento

\begin{tabular}{|c|c|c|c|c|c|}
\hline $\begin{array}{l}\text { Bienestar } \\
\text { subjetivo }\end{array}$ & $X$ & $D E$ & $\alpha$ & $\begin{array}{c}\text { Valor } \\
\text { propio }\end{array}$ & $\begin{array}{c}\% \text { de } \\
\text { varianza }\end{array}$ \\
\hline General & 2.36 & 3.42 & .72 & 2.50 & 50.05 \\
\hline Familiar & 2.35 & 4.24 & .86 & 3.31 & 66.23 \\
\hline Escolar & 2.23 & 3.55 & .76 & 2.64 & 52.86 \\
\hline Trabajo & 2.44 & 4.14 & .77 & 2.67 & 53.43 \\
\hline Relación de pareja & 2.07 & 3.82 & .83 & 3.00 & 60.10 \\
\hline \multicolumn{6}{|l|}{ Expectativas } \\
\hline General & 3.31 & 2.02 & .53 & 2.05 & 41.12 \\
\hline Familiar & 3.44 & 1.72 & .61 & 1.84 & 30.67 \\
\hline Escolar & 3.45 & 1.70 & .64 & 1.94 & 48.73 \\
\hline Trabajo & 3.47 & 2.56 & .71 & 2.65 & 44.23 \\
\hline Relación de pareja & 3.78 & 1.03 & .59 & 1.34 & 22.36 \\
\hline \multicolumn{6}{|l|}{ Autoeficacia } \\
\hline General & 2.04 & 2.12 & .58 & 1.85 & 35.88 \\
\hline Familiar & 2.29 & 2.91 & .56 & 2.28 & 15.97 \\
\hline Escolar & 1.58 & 2,47 & .73 & 2.50 & 50.14 \\
\hline Trabajo & 1.81 & 3.26 & .82 & 3.03 & 60.78 \\
\hline Relación de pareja & 2.07 & 1.93 & .39 & 1.39 & 27.87 \\
\hline \multicolumn{6}{|l|}{ Motivación de logro } \\
\hline General & 4.39 & 1.88 & .57 & 1.25 & 5.44 \\
\hline Familiar & 4.38 & 1.42 & .77 & 1.04 & 4.53 \\
\hline Escolar & 4.05 & 4.30 & .81 & 1.70 & 7.42 \\
\hline Trabajo & 4.48 & 2.27 & .82 & 3.15 & 13.73 \\
\hline Relación de pareja & 3.78 & 4.90 & .84 & 6.52 & 28.37 \\
\hline \multicolumn{6}{|c|}{ Individualismo-Colectivismo } \\
\hline Egoísmo & 3.61 & 9.69 & .72 & 7.19 & 6.61 \\
\hline Independencia & 2.40 & 3.79 & .38 & 3.12 & 17.47 \\
\hline Individualismo & 2.93 & 4.60 & .56 & 1.95 & 22.11 \\
\hline
\end{tabular}

El análisis de factores se realizó vía componentes principales, con rotación Varimax.

La confiabilidad se obtuvo con la formula alfa de Cronbach.

La edad correlacionó con el bienestar subjetivo en la relación de pareja $(\mathrm{r}=-.11, p<.05)$, autoeficacia en el trabajo $(\mathrm{r}=-.21, p<.05)$ e independencia $(\mathrm{r}=$ $-.12, p<.05)$.

Se hicieron pruebas de diferencia de medias a partir de la variable sexo. Los resultados indican que sólo en la variable de nivel de aspiración familiar se presentan diferencias entre hombres y mujeres, con una media de 4.01 para hombres y una media de 3.98 para las mujeres $\left(\mathrm{t}_{402}=6.8, p<.05\right)$. 


\section{Bienestar general}

Los resultados de la regresión lineal se presentan a continuación a partir de las diferencias obtenidas por sexo. En la muestra de los hombres el modelo explica el $68.3 \%$ de la varianza $\left(R^{2}\right.$ ajustada) sobre la variable dependiente de satisfacción con la vida en general. La variable que influye directamente es la satisfacción con la comunicación familiar $(\mathrm{B}=.833)$. Ninguna de las otras variables logra explicar. El modelo es consistente y confiable $(\mathrm{F} 1,32=70.01 p<.05)$. En el caso de las mujeres tenemos algo distinto. Aunque el modelo es consistente y confiable (F3,36 $=52.14 p<.05)$ y explica el $36.4 \%$ de la varianza (R2 aj), las variables que influyen son la satisfacción con la comunicación familiar $(\mathrm{B}=.81)$, la satisfacción con la vida familiar $(\mathrm{B}=.46)$ y la autoeficacia en el trabajo $(\mathrm{B}=.21)$.

\section{Bienestar por ámbitos particulares}

Respecto a la variable de satisfacción con la vida familiar, tenemos que en los hombres sólo influye la satisfacción con la comunicación familiar con un valor de $\mathrm{B}=.63$. Este modelo es consistente y confiable $(\mathrm{F} 1,32=21.19 p<.05)$ y explica el $64 \%$ de la varianza (R2 aj). En el caso de las mujeres el modelo es explicado por el $91 \%$ de la varianza (R2 aj). Las variables que impactan de manera positiva son la satisfacción con la comunicación familiar $(\mathrm{B}=.76)$, la autoeficacia escolar $(\mathrm{B}=.29)$, el nivel de aspiración en la relación de pareja $(B=.26)$, las expectativas generales $(B=1.31)$ y la autoeficacia en la relación de pareja $(B=.15)$. Las variables satisfacción de apoyo familiar $(B=-89)$, las expectativas de trabajo $(\mathrm{B}=-.24)$, la autoeficacia general $(\mathrm{B}=-.22)$, la independencia $(B=-14)$ y el nivel de aspiración escolar $(B=-16)$ impactan de manera negativa.

En relación con la variable dependiente satisfacción escolar, las variables que influyen en las adolescentes son: la satisfacción en el trabajo $(\mathrm{B}=.34)$ y las expectativas escolares $(B=-.36)$. Cabe señalar que esta última influye de manera negativa. Este modelo explica el $20 \%$ de la varianza (R2 aj), es un modelo consistente y confiable (F2,36 $=5.52 P<.05)$. El modelo que predice a la muestra de hombres nos muestra que las variables de satisfacción en el trabajo $(B=.68)$, la satisfacción en la comunicación familiar $(\mathrm{B}=.41)$, y la autoeficacia escolar $(\mathrm{B}=.31)$, influyen directamente en la satisfacción en el trabajo y sólo la variable de autoeficacia en la comunicación familiar impacta de manera negativa $(B=-.30)$. Dicho modelo explica el $72 \%$ de la varianza (R2 aj). Es un modelo consistente y por lo tanto, confiable (F4,32 $=22.04 p<.05$ ).

Las variables de satisfacción escolar $(\mathrm{B}=.68)$, y nivel de aspiración con la vida en general $(\mathrm{B}=-.29)$ influyen en la satisfacción laboral para el caso de los adolescentes. El modelo explica el $52 \%$ de la varianza ( $R 2$ aj), es un modelo consistente y confiable (F2,32 $=18.42 p<.05)$. Para las adolescentes tenemos que las expectativas de trabajo $(\mathrm{B}=.38)$, la satisfacción con sus estudios $(\mathrm{B}=.32)$ y el nivel de aspiración en la vida en general $(B=-.44)$ influyen en la satisfacción laboral, aunque esta última lo hace de manera negativa. Este modelo logra explicar el $36.4 \%$ de la varianza (R2 aj). Es un modelo confiable (F3,36 = $7.86 \mathrm{P}<.05)$.

Por último, tenemos la variable dependiente de satisfacción en la relación de pareja, en donde tenemos que, para los hombres, las variables que influyen son: la satisfacción laboral $(\mathrm{B}=.59)$, la satisfacción del apoyo por parte de la familia $(\mathrm{B}=.32)$, $\mathrm{y}$ las expectativas generales $(\mathrm{B}=.33)$, además de las variables de nivel de aspiración en relación con el reconocimiento social $(\mathrm{B}=-.39)$ y las expectativas escolares $(\mathrm{B}=-.27)$, las cuales impactan de manera negativa. El modelo explica el $64 \%$ de la varianza (R2 aj). Es un modelo confiable y constante (F5,32 $=12.57 p<$ $.05)$. En la muestra de las adolescentes tenemos algo diferente, ya que solo la variable de satisfacción laboral $(\mathrm{B}=.37)$ predice la satisfacción en la relación de pareja. El modelo explica solo el $12 \%$ de la varianza (R2 aj). Es un modelo confiable y constante $(\mathrm{F} 1,36=5.83, p<.05)$.

\section{Discusión}

En este estudio buscamos en primer lugar desarrollar una escala para evaluar el alcance de las expectativas de una muestra de adolescentes de la Ciudad de México. El instrumento mostró evidencia de una aceptable validez factorial, explicando entre el 31 y el $48 \%$ de la varianza, pero los coeficientes de confiabilidad no fueron del todo satisfactorios (alfas en un rango entre .53 y .71). Esto puede deberse a tres factores: a) al empleo de seis reactivos para cada área que puede haber resultado un número insuficiente; $b$ ) al formato de respuesta, el cual tomó como inspiración instrumentos como el BDI (Beck, 1961) que incluyen en cada reactivo cuatro alternativas en orden creciente 
del rasgo o indicador a medir; y c) a la variabilidad de la muestra, donde debe recordarse que incluimos jóvenes de dos escenarios educativos claramente diferenciados. En todo caso, el funcionamiento de la escala amerita una investigación posterior sobre estas líneas de desarrollo, dado que no existe una escala equivalente y la que aquí utilizamos muestra cierta validez de contenido pero baja confiabilidad, y por tanto no puede usarse para calificación individualizada.

Cabe hacer notar el alto grado de expectativas de los jóvenes en todos los ámbitos, destacando el de relación de pareja donde, de cuatro puntos máximos, los participantes obtuvieron 3.78 . Por ejemplo, $57 \%$ de la muestra espera tener una relación sentimental excelente y $39 \%$ buena. Sin embargo, esos niveles de expectativas disminuyen cuando se inquiere sobre la vida en general, con el promedio más bajo de esta escala (3.31). Quizá el mejor ejemplo de esto es que sólo el $38 \%$ de los jóvenes esperan estar muy satisfechos con su vida en 10 años y el mismo porcentaje espera haber logrado las metas que se haya planteado para ese momento.

Puede ser que la explicación de este dato vaya por el lado de lo abstracto e indeterminado de una pregunta sobre expectativas generales, lo que explicaría el porqué de la tendencia observada entre los ámbitos, o bien, que en realidad exista conciencia de la dificultad de plantear metas muy ambiciosas (que no lo serian para otras generaciones) dado el contexto socioeconómico actual.

Por su parte, las escalas de bienestar subjetivo, motivación de logro y en menor medida la de autoeficacia y de colectivismo-individualismo, parecen comportarse adecuadamente para esta muestra. Los jóvenes encuestados muestran un nivel de autoeficacia de alto a moderado, mismo rango observado para el bienestar subjetivo. De nuevo, estos atributos personales varian con el ámbito evaluado, con un mejor nivel de control percibido y de satisfacción en el área de relaciones de pareja, espacio donde probablemente perciban tener mayores elementos para dirigir el destino e intervenir en el destino de la relación, pero paradójicamente más bajo en términos de las relaciones familiares que esperan establecer.

Junto con lo anterior, esta generación muestra un acuerdo fundamental con valores que ponen a las necesidades y metas individuales antes que las grupales, sea a través de la autoafirmación (individualismo) e incluso con rasgos de egocentrismo (egoísmo). $\mathrm{Si}$ esta es una tendencia debida a la etapa de la vida en que fueron entrevistados o pertenezca a una matriz cultural y aun momento histórico de más largo alcance, esta es una pregunta que queda por contestar en investigaciones de carácter longitudinal.

A partir de la construcción teórica realizada en este estudio encontramos que las variables consideradas sí están relacionadas de manera significativa con el bienestar subjetivo. La relación teórica que esperábamos encontrar entre las expectativas y el bienestar subjetivo, aunque no es muy marcada, si aparece como relevante en éste, aunque sólo sea para algunos ámbitos: ámbito escolar, de relación de pareja y de trabajo, lo cual nos lleva a sugerir que estas variables sean estudiadas en otros trabajos posteriores.

Los constructos teóricos de autoeficacia y motivación al logro resultaron ser variables importantes, ya que, si se perciben un alto nivel de eficacia y un alto nivel de aspiraciones, estos impactarán de la misma manera en el bienestar subjetivo.

\section{Referencias}

Affleck, M. \& cols. (1989). The Influence of Gender Role Attitudes on Life Expectations of College Students. Youth and Society, 20(3), 307-19.

Andrade, P. \& Reyes, I. (1996). Locus de Control y Orientación al Logro en Hombres y Mujeres. Revista de Psicologia Social y Personalidad, I2(1-2), 75-84.

ANUIES (2000). La educación superior en el siglo xxi. Lineas estratégicas de desarrollo. Una propuesta de la ANUIEs. México: ANuIBs.

Bandura, A. (1986). Social foundations of thought and action: A social cognitive theory. Englewood Cliffs, Nueva Jersey: Prentice-Hall.
Bandura, A. (Ed.) (1999). Auto-eficacia: Cómo afrontamos los cambios de la sociedad actual. Bilbao: Desclée De Brouwer.

Beck, A., Ward, C., Mendelson, M., Mock, J. \& Erbaugh, J. (1961). An inventory for measuring depression. Arch Gen Psychiatry, 4, 53-63.

Cantor, N., Norem, J. \& Brower, A. (1987). Life tasks, selfconcept ideals and cognitive strategies in a life transition. Journal of Personality and Social Psychology, 53(6), 1178-1191.

Cambell, A., Converse P. \& Rodgers, W. (1976). The quality of American life: Perceptions, evaluations and satisfactions. Nueva York: Russell Sage. 
Correa, F., Contreras, C., Ramírez, A. \& López, E. (2002). Dimensiones del individualismo-colectivismo en México: Un estudio exploratorio. Revista de la Psicología Social en México, 9, 553-559.

Costa, P. \& McCrae, R. (1980). Influence of extraversion and neuroticism on subjetive well being: Happy and unhappy people. Journal of Personality and Social Psychology. 38(4), 668-678.

Díaz Morales, J. \& Sánchez-López, M. (2001). Relevancia de los estilos de personalidad y las metas personales en la predicción de la satisfacción vital. Anales de Psicologia, 17(2), 151-158.

Diener, E. (1984). Subjective well-being. Psychological Bulletin, 95, 542-575.

Diener, E. (1984). Culture and subjective well-being. Annual Review of Psychology, 22.

Diener, E., Oishi, S. \& Lucas, R. (2003). Personality, culture, and subjective well-being: Emotional and Cognitive Evaluations of Life. Annual Review of Psychology, 54, 403-425.

Espinoza, R. \& Reyes Lagunes, 1. (1992). La evitación del éxito y la orientación de logro ¿un rasgo de la personalidad? La Psicología Social en México, 4, 388-393.

Grau, J.A. (2003). Calidad de vida y salud: Problemas actuales en su investigación. Boletin Latinoamericano de Psicología de la Salud. Disponible en Asociación Latinoamericana de Psicologia de la Salud (Alapsa). http:// www.alapsa.org/boletin/art02.html; consultado el 9 de noviembre de 2003.
Instituto Mexicano de la Juventud (Imeju) (2000). Resultados preliminares de la encuesta nacional de la juventud. México: IMJ.

López, I. \& Páez, D. (1996). Bienestar subjetivo en el ambiente acústico: afectos positivos y afectos negativos. $R e$ vista de Psicologia Social, 2, 103-107.

McClelland, D.C. (1985). Estudio de la motivación humana. Madrid: Narcea.

Muneé, F. (1989). Entre el individuo y la sociedad. Madrid: Eudeba.

Palomar, J. \& Pérez, A. (2001). Aspectos que impactan el bienestar de los pobres. Psicologia Iberoamericana, 9(2), 66-67.

Settersten, R. \& Mayer, K. (1997). The measurement of age, Age structuring and the life course. Annual Review of Sociology, 23, 233-261.

Tapia, V.A. (1998). Los hijos del dios forastero: Cultura y religión en el México de hoy. Revista de Psicología Social en México, 7, 35-40.

Triandis, H.C. (1990). Aproximaciones teóricas y metodológicas al estudio del individualismo y colectivismo. $R e$ vista de Psicologia Social y Personalidad, 6(1-2), 29-38.

Triandis, H.C. (1994). Cultura: El nuevo enfoque en psicologia. Revista de Psicología Social y Personalidad, 10(1), 1-16.

Valenzuela, M., Díaz-Loving, R. \& Manjarrez, J.O. (1996). Diferencias entre las variables sociales y demográficas en el bienestar subjetivo. La Psicologia Social en México, 6, 425-431.

\section{Anexo. Escala de Expectativas}

\section{Ambito general}

1. En 10 años en mi vida en general, espero estar:

a) Insatisfecho con mi vida.

b) Algo satisfecho con mi vida.

c) Muy satisfecho con mi vida.

d) Completamente satisfecho con mi vida.

2. En relación con mi vida en general, en 10 años espero tener una vida:

a) Peor a la que ahora tengo.

b) Igual.

c) Mejor.

d) Mucho mejor a la que ahora tengo.
3. Respecto a mis metas personales, en 10 años espero haber logrado:

a) Ninguna de mis metas.

b) Algunas de mis metas.

c) Muchas de mis metas.

d) Todas mis metas.

4. En relación con la opinión de los demás, en 10 años, espero que la gente tenga una:

a) Mala opinión de mí.

b) Opinión regular.

c) Buena opinión.

d) Excelente opinión de mí. 
5. En general, ¿cómo ves tu futuro próximo?
a) Con mucho pesimismo.
b) Con algo de pesimismo.
c) Con algo de optimismo.
d) Con mucho optimismo.

\section{Ambito familiar}

6. En términos de formar una familia, en 10 espero encontrarme:

a) Sin planes para formar una familia.

b) Estar formando una familia.

c) Tener una familia formada (es decir con esposo/a e hijos).

d) Separado de mi esposo/a e hijos.

7. En cuanto al reconocimiento que pueda tener de mis familiares y amigos, en 10 años espero ser:

a) Poco reconocido(a) por ellos.

b) Algo reconocido(a).

c) Reconocido(a).

d) Muy reconocido(a) por ellos.

8. En cuanto a la comunicación con mi familia, en 10 años espero tener una:

a) Mala comunicación familiar.

b) Comunicación familiar regular.

c) Buena comunicación familiar.

d) Excelente comunicación familiar.

9. Respecto a la familia que puedo tener, en 10 años espero:

a) No tener una familia.

b) Tener una familia peor a la que ahora tengo.

c) Tener una familia igual.

d) Tener una familia mejor a la que ahora tengo.

10. En cuanto al apoyo familiar, en 10 años espero:

a) No tenc apoyo familiar.

b) Tener algo de apoyo familiar.

c) Que mi familia me apoye lo suficiente.

d) Tener todo el apoyo familiar posible.
11. En relación con las decisiones que se tomen en mi familia, en 10 años espero:

a) Se tomen malas decisiones.

b) Las decisiones que se tomen sean regulares.

c) Mi familia tome buenas decisiones.

d) Sean muy buenas las decisiones que se tomen en mi familia.

\section{Ambito escolar}

12. En relación con mis estudios en general, en 10 años espero:

a) No haber estudiado.

b) Tener una carrera técnica (secretaria o mecánico, por ejemplo).

c) Tener un titulo universitario.

d) Estudiar un posgrado dentro o fuera del país.

13. Respecto a la satisfacción con mis estudios, en 10 años espero encontrarme:

a) Insatisfecho con los estudios que tenga.

b) Algo satisfecho.

c) Muy satisfecho.

d) Completamente satisfecho con los estudios que tenga.

14. En 10 años espero que mi preparación académica sea:

a) Peor a la que shora tengo.

b) Igual.

c) Mejor.

d) Mucho mejor a la que ahora tengo.

15. En cuanto al promedio de calificaciones de mis estudios en general, en 10 años espero:

a) No haber terminado mis estudios.

b) Terminar mis estudios con un bajo promedio de calificaciones.

c) Terminar mis estudios con un buen promedio.

d) Terminar mis estudios con un reconocimiento (por ej. un diploma) debido a mi promedio. 


\section{Ambito de trabajo}

16. En relación con mi trabajo en general, en 10 años espero:
a) No tener trabajo.
b) Tener un trabajo seguro
c) Tener un trabajo eventual.
d) Tener un trabajo para toda la vida.

17. En cuanto al puesto en mi trabajo, en 10 años espero:

a) No tener trabajo.

b) Ser empleado (por ejemplo, obrero/a), oficinista, etc.)

c) Tener personas a mi cargo.

d) Ser empresario.

18. Con relación al prestigio social de mi trabajo, en 10 años espero que mi trabajo:

a) No tenga un prestigio social.

b) Sea poco importante.

c) Sea lo suficientemente importante.

d) Tenga un excelente prestigio social.

19. En cuanto a mi situación económica en general, en 10 años espero:

a) Tener una seguridad económica mala.

b) Vivir con una seguridad económica regular.

c) Lograr una seguridad económica buena.

d) Tener una excelente seguridad económica.

20. Respecto a la utilidad de mi trabajo, en 10 años espero que mi trabajo:

a) No sea útil a otras personas.

b) Sea poco útil.

c) Sea lo suficientemente útil.

d) Sea de gran utilidad a otras personas.

21. En relación con el reconocimiento en el trabajo, en 10 años espero:

a) No ser reconocido por mis capacidades personales y profesionales.

b) Ser poco reconocido.

c) Ser lo suficientemente reconocido.

d) Ser muy reconocido por mis capacidades personales y profesionales.

\section{Ambito de relación de pareja}

22. En 10 años, espero que mi relación de pareja en general, sea:
a) Mala.
b) Regular.
c) Buena.
d) Excelente.

23. En términos de satisfacción de necesidades emocionales (por ej., cariño, apoyo, comprensión, etc.), en 10 años espero encontrarme con mi pareja:
a) Nada satisfecho(a).
b) Poco satisfecho(a).
c) Regularmente satisfecho(a).
d) Muy satisfecho(a).

24. Respecto al apoyo de mi pareja en general, en 10 años espero:

a) No contar con el apoyo de mi pareja.

b) Tener un poco de su apoyo

c) Que mi pareja me apoye lo suficiente.

d) Que mi pareja me apoye completamente.

25. En cuanto al apoyo económico de mi pareja, en 10 años espero:
a) No contar con ningún apoyo económico.
b) Me apoye algo.
c) Tener el suficiente apoyo de mi pareja.
d) Que mi pareja me apoye completamente.

26. En cuanto a la toma de decisiones en mi relación de pareja, en 10 años espero:

a) Que otras personas tomen las decisiones.

b) Ser yo quien tome las decisiones.

c) Que mi pareja tome las decisiones

d) Que ambos tomemos las decisiones.

27. En relación con las tareas domésticas, en 10 años, mi pareja me apoye en:

a) Ninguna de las tareas domésticas.

b) Algunas de las tareas

c) En muchas tareas domésticas.

d) En todas las tareas domésticas. 